\title{
TLC599 in patients with osteoarthritis of the knee: a phase lla, randomized, placebo- controlled, dose-finding study
}

\author{
David J. Hunter ${ }^{1,2}$, Chi-Ching Chang ${ }^{3,4}$, James Cheng-Chung Wei ${ }^{5,6,7}$, Hsiao-Yi Lin ${ }^{8,9}$, Carl Brown ${ }^{10}$, Tien-Tzu Tai ${ }^{10}$, \\ Chih-Feng $\mathrm{Wu}^{10}$, Wing Chia-Ming Chuang ${ }^{10}$ and Sheue-Fang Shih ${ }^{10^{*}}$
}

\begin{abstract}
Background: Corticosteroid injection for knee osteoarthritis is limited by its modest duration of treatment effect. The liposome formulation of dexamethasone sodium phosphate (TLC599) was developed for the sustained relief of osteoarthritis pain. This clinical study was conducted to evaluate the efficacy and safety of TLC599 at two dose levels in patients with knee osteoarthritis.
\end{abstract}

Methods: A randomized, double-blinded, placebo-controlled study was conducted in 75 patients with osteoarthritis of the knee from 13 study centers. Patients were randomized and administered a single intra-articular injection of TLC599 or placebo and assessed for efficacy and safety for 24 weeks. Patient-reported outcomes included the Western Ontario and McMaster Universities Arthritis (WOMAC) Index for pain and function and visual analog scale for pain.

Results: TLC599 at 12 mg demonstrated significantly greater reduction in WOMAC pain through 12 weeks (least squares (LS) mean difference $=-0.37, p=0.0027$ ) and through 24 weeks (LS mean difference $=-0.35, p=0.0037$ ) when compared to placebo. TLC599 $12 \mathrm{mg}$ also exhibited significantly greater improvement in function when compared to placebo at 24 weeks (LS mean difference $=-0.26, p=0.0457$ ). TLC599 $18 \mathrm{mg}$ did not significantly improve pain or function in comparison with placebo. The use of acetaminophen during the study was less in both TLC599 groups in comparison with placebo. No major or unexpected safety issues were reported.

Conclusions: In participants with symptomatic knee osteoarthritis, TLC599 is a well-tolerated treatment that reduces pain and improves function for up to 24 weeks, a longer duration than that reported for existing IA treatments.

Trial registration: ClinicalTrials.gov, NCT03005873. Registered on 29 December 2016

Keywords: Knee osteoarthritis, Glucocorticoid, Pain, WOMAC, VAS

\section{Background}

Osteoarthritis (OA) is a rheumatic musculoskeletal disorder with a high prevalence and affects more than 200 million people worldwide [1]. As the most common form of arthritis, OA is the leading cause of chronic disability

${ }^{*}$ Correspondence: yvonne@tlcbio.com

${ }^{10}$ Taiwan Liposome Company, Ltd., 2F, No. 3 Yuanqu St., Nangang Dist., Taipei City 115, Taiwan

Full list of author information is available at the end of the article and reduced activity in elders [2]. The Osteoarthritis Research Society International (OARSI) also describes $\mathrm{OA}$ as a serious disease [3]. OA affects the synovial joints including those of the knee, hands, hip, and spine, and patients with knee OA accounts for a large proportion of OA cases. In particular, knee OA is characterized by articular and subchondral bone cartilage degradation and osteophyte formation, leading to joint pain, impairment in movement, and reduction in physical function or daily activities [4]. It was estimated that 14 million people in original author(s) and the source, provide a link to the Creative Commons licence, and indicate if changes were made. The images or other third party material in this article are included in the article's Creative Commons licence, unless indicated otherwise in a credit line to the material. If material is not included in the article's Creative Commons licence and your intended use is not permitted by statutory regulation or exceeds the permitted use, you will need to obtain permission directly from the copyright holder. To view a copy of this licence, visit http://creativecommons.org/licenses/by/4.0/. The Creative Commons Public Domain Dedication waiver (http://creativeco mmons.org/publicdomain/zero/1.0/) applies to the data made available in this article, unless otherwise stated in a credit line to the data. 
the US have symptomatic OA [5], and around 7.7 million have advanced radiographic knee OA, as characterized by a Kellgren-Lawrence (KL) grading score of 3-4; almost half are between 45 and 64 years of age.

Treatments for knee OA primarily involve a combination of exercise and lifestyle modification, pharmacological treatment, over-the-counter supplements, and surgical joint replacement. Non-surgical treatments are usually effective for patients in the early stages of OA (KL grades 1 to 3), while surgical treatment with joint replacement is frequently a choice for patients with endstage knee OA. Pharmacological treatments, such as acetaminophen, non-steroidal anti-inflammatory drugs (NSAIDs), cyclooxygenase-2 inhibitors, corticosteroid injections, and tramadol are used by OA patients for symptom relief $[6,7]$. In particular, there is an increasing trend in opioid prescription to treat OA pain, with poor patient satisfaction, and increased morbidity and mortality [8]. Intraarticular (IA) corticosteroids are recommended as a standard treatment for OA of the knee, though treatment effect is of modest duration, often for only 2 to 4 weeks [9]. Furthermore, repeat injections are normally limited to 4 injections annually $[10,11]$, and it was shown that repeated quarterly IA corticosteroids in knee OA for 2 years were associated with more cartilage loss than saline injection [12]. A non-opioid treatment that could effectively reduce pain and provide sustained pain relief could fulfill a large unmet need.

TLC599 is a liposome formulation of dexamethasone sodium phosphate (DSP), a water-soluble and potent glucocorticoid. It was developed with the BioSeizer platform technology [13] to prolong the local residence of DSP in the joint space, with the potential to reduce injection frequency and risk, while maximizing clinical benefit. The objective of the current clinical study was to evaluate the efficacy and safety of a single IA injection of TLC599 over a period of 24 weeks in participants with OA pain of the knee.

\section{Methods}

The aim of this study was to evaluate the treatment efficacy of different test doses of TLC599 in patients with symptomatic knee OA. The study was a randomized, double-blinded, placebo-controlled phase IIa clinical trial (ClinicalTrials.gov no.: NCT03005873) to test two TLC599 doses (12 $\mathrm{mg}$ and $18 \mathrm{mg}$ DSP) compared to placebo in participants with knee OA. The study protocol, all study protocol amendments, investigator's brochure, informed consent form, and any other relevant documents were reviewed and approved by an independent ethics committee (IEC) or institutional review board (IRB) at each study center. The study was conducted in accordance with the approved protocol, the ethical principles derived from international guidelines including the Declaration of Helsinki (2013), International Council for Harmonisation, Good Clinical Practice Guidelines, and applicable laws and regulations. An informed consent form was signed by the participant or representative before they entered the study. Thirteen study centers (5 in Australia and 8 in Taiwan) participated in the study, and approximately 72 participants were planned for enrollment. The study was conducted between May 2017 and July 2018.

\section{Eligibility}

Key inclusion criteria for eligible subjects included males or females $\geq 50$ years of age with OA associated symptoms for $\geq 6$ months, OA confirmation based on the American College of Rheumatology Criteria for Classification of Idiopathic OA of the knee, a radiographic KL grade 2 or 3 for knee OA severity, and a visual analog scale (VAS) self-reported pain score between 5.0 and 9.0 (out of 10) in the study knee. Key exclusion criteria included the use of systemic corticosteroids within 30 days prior to dosing; glucosamine, chondroitin, or dietary supplement with unstable dose or frequency within 4 weeks before screening; IA corticosteroid, hyaluronic acid, and other IA injection within 3 months prior to screening; chemotherapeutic or systemic immunosuppressant agents for inflammatory diseases; investigational agents within 6 months prior to screening; and concurrent use of anticoagulants. Participants with any use of new rehabilitation or exercise program within the specified time frame before or during screening were also excluded. Also, the use of prohibited medications other than acetaminophen and oral NSAIDS within $48 \mathrm{~h}$ and 7 days, respectively, prior to dosing was excluded. Other exclusion criteria associated with participant's health condition included autoimmune diseases, IA bleeding, infective arthritis or gout attack, amputation of the lower limb, unstable knee joint, acute injury to the study knee in the prior 6 months, any surgery or arthroscopy in the study knee in the prior 12 months, acute infection or infectionrelated inflammation (non-study knee), skin lesion or breakdown at the injection site, body mass index $>40$ $\mathrm{kg} / \mathrm{m}^{2}$, low platelet count or blood coagulation disorder, history of acquired or congenital immunodeficiency diseases, concurrent or uncontrolled infectious disease, history of treated malignancy with disease free for $\leq$ 5 years, stroke or myocardial infarction, uncontrolled and unstable concurrent medical or psychiatric illness, allergy or hypersensitivity to the study drug, pregnancy, and pre-defined laboratory abnormalities. 


\section{Study treatment, randomization, and concealment}

For each participant, one knee was selected and defined as the study knee to receive study treatment. The study knee was determined based on the presentation of symptoms associated with OA for 6 months prior to screening visit with an equal or higher VAS pain score than the non-study knee. If the VAS score for both knees diagnosed as OA was equal, the study knee was selected by the investigator with reason documented. Eligible participants were randomized in a ratio of 1:1:1 (block size $=6$ ) using an interactive web response system (IWRS, Cenduit Interactive Response Technology), with stratification based on bilateral (VAS pain score $\geq$ 3 in the non-study knee) or unilateral (VAS pain score $<3$ in the non-study knee) knee pain, to receive a single IA injection of one of the following three blinded treatments at baseline: (1) TLC599 at $12 \mathrm{mg}$ DSP with 100 umol phospholipid (PL) (TLC599 $12 \mathrm{mg} ; 1.0 \mathrm{~mL}$ ), (2) TLC599 at $18 \mathrm{mg}$ DSP with $150 \mu \mathrm{mol}$ PL (TLC599 18 $\mathrm{mg}$; $1.5 \mathrm{~mL}$ ), or (3) placebo (1.5 mL normal saline) at day 0 .

The study included blinded and unblinded teams; only the unblinded team responsible for study drug injection had access to the study drug identity. The study treatments were stored in boxes attached with tamperevident tape while the dosing syringe was wrapped with non-transparent tape to ensure the blindness of the participants. The unblinded injector performed the study drug injection using a 21-gauge needle under aseptic conditions.

Following injection of the study medication, only acetaminophen up to $3 \mathrm{~g}$ /day was permitted as pain rescue medication during the study but was not to be used within $48 \mathrm{~h}$ prior to efficacy assessment at scheduled visits. Other pain medications including NSAIDs and opioids were not permitted.

\section{Outcome measures and follow-up visits}

Efficacy assessments were based on measurement outcomes at the scheduled study visits using patientreported questionnaires, including the Western Ontario and McMaster Universities Arthritis Index (WOMAC)-Pain and WOMAC-Function subscales, and patient-reported pain on a VAS. The WOMAC is a selfadministered 24-item scale (each on a Likert scale of 0 to 4 , with higher scores indicating worse symptoms, divided into subscales of pain, stiffness, and function; average score in each subscale was calculated for analysis). Eligible subjects were scheduled for study visits on day 0 (baseline), day 3, and weeks 1, 4, 8, 12, 16, 20, and 24. The outcome measures with participant-administered questionnaires were conducted at each study visit.
The primary endpoint was the change from baseline in WOMAC-Pain score through week 12 . Secondary analyses included change from baseline in WOMAC-Pain, WOMAC-Function, and VAS pain scores at various time points up to week 24 , as well as the proportion of clinically durable responders (defined as $>30 \%$ pain reduction as measured by the WOMAC-Pain scale in every subsequent post-dosing visit). The EuroQol-5 Dimension (EQ-5D) for assessing the quality of life was also completed by the participants at the scheduled visits. Daily acetaminophen consumption (prohibited within $24 \mathrm{~h}$ prior to the study visits) was reported by the participants using a paper diary.

Safety assessment of the study drugs was based on the collection of adverse events, physical examination, clinical chemistry and hematology, urinalysis, 12-lead electrocardiogram (ECG), hemoglobin A1c (HbA1c), blood cortisol, and magnetic resonance imaging (MRI) of both knees.

\section{Sample size}

No formal sample size calculation was performed for this study, as this was the first randomized, blinded, and placebo-controlled study conducted with the study drug TLC599. It was anticipated approximately 20 participants in each study group would complete 24 weeks of followup after study drug dosing. A drop-out rate of $15 \%$ was expected, requiring approximately 72 subjects enrolled in the study.

\section{Statistical analyses}

Efficacy analyses were performed using the modified intent-to-treat population (mITT) data, which included all randomized participants who received a complete dose of TLC599 or placebo and had at least 1 complete efficacy evaluation after study drug dosing. The changes from baseline in WOMAC-Pain, WOMAC-Function, and VAS pain scores were analyzed using a mixedeffects model for repeated measures (MMRM) with restricted maximum likelihood estimation. The model included factors of treatment, visit (as a categorical variable), and baseline value as fixed factors; center as a random factor; and treatment-by-visit as interaction terms. Changes from baseline at each time point, and for intervals through defined time points, as well as differences between treatment groups and placebo, were estimated using this methodology. Clinically durable responders were analyzed using a logistic regression model including treatment group and baseline value of WOMAC-Pain. The least squares means and 2-sided 90\% confidence intervals were reported, and all statistical assessments were conducted 1-sided and evaluated at the $5 \%$ level of 
significance; analyses were performed using SAS version 9.4.

The safety data were summarized for all participants who received any dose of the study drug (safety population). No interim analysis was planned in this study, and all analyses were conducted after the database lock per statistical analysis plan.

For primary and secondary efficacy analyses, missing data was not imputed. All data collected was included in the assessment of patient safety, while missing or incomplete AE data assumed the greatest relationship to study drug and/or severity.

\section{Results}

\section{Demographic characteristics}

A total of 148 participants were screened for participation and there were 72 screen failures. The 76 participants in the intent-to-treat population (all randomized patients) included 26 participants assigned to placebo, 26 to TLC599 $12 \mathrm{mg}$, and 24 to TLC599 $18 \mathrm{mg}$ (Fig. 1). One participant assigned to placebo was found to be ineligible after randomization, was withdrawn prior to receiving study treatment, and was not included in the safety and mITT populations.
In the safety population $(n=75)$, the majority $(60.0 \%)$ of participants were aged 50 to 65 years, and the mean age was 63.9 years. There were more female (66.7\%) than male $(33.3 \%)$ participants. Study participants were mostly Asian (from Taiwan, 50.0\%) or Caucasian (from Australia, 50.0\%). Demographic characteristics were generally balanced among the treatment groups, although there were somewhat greater proportions of males and participants with KL scores of grade 2 in the TLC599 12 mg group (Table 1).

\section{Efficacy}

Based on the mITT population $(n=75)$, participants receiving a single IA dose of TLC599 $12 \mathrm{mg}$ demonstrated a statistically significant improvement in WOMAC-Pain score compared to placebo through week $12(p=0.0027)$, meeting the study's primary endpoint (Fig. 2; Table 2). Further, the improvement was persistent, with statistical superiority compared to placebo, reflecting a sustained duration of pain control through week 24 . Improvements in WOMAC-Pain with TLC599 $18 \mathrm{mg}$ did not reach statistical significance compared with placebo for any time interval.

Using a landmark analysis at each assessment visit against baseline, participants receiving TLC599

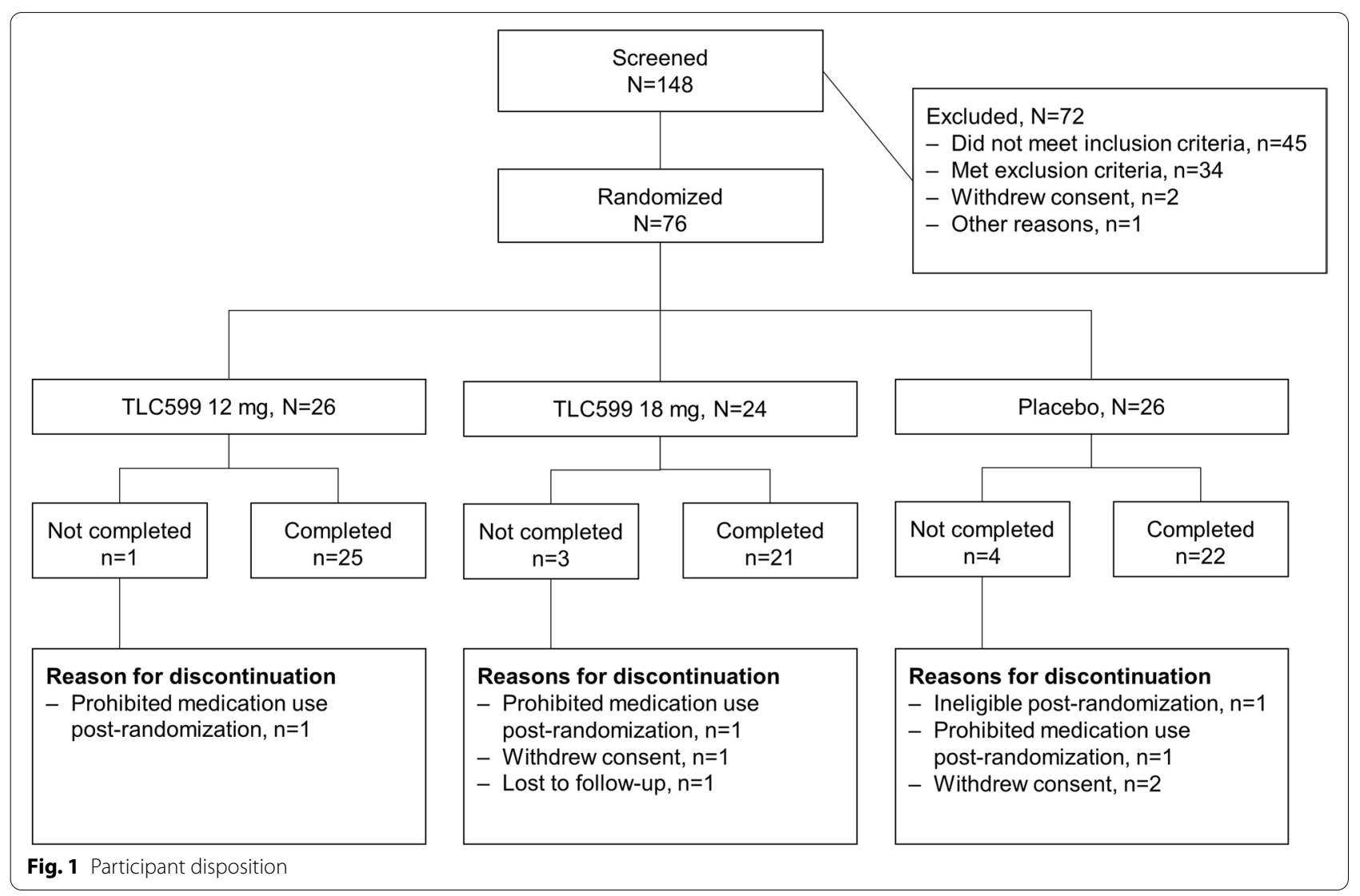


Table 1 Baseline demographic and clinical characteristics (safety population)

\begin{tabular}{|c|c|c|c|c|}
\hline Characteristic & Statistics & Placebo $(n=25)$ & TLC599 $12 \mathrm{mg}(n=26)$ & TLC599 $18 \mathrm{mg}(n=24)$ \\
\hline Age (years) & Mean (SD) & $64.8(8.45)$ & $63.9(9.07)$ & $62.9(8.80)$ \\
\hline \multicolumn{5}{|l|}{ Gender } \\
\hline Male & $n(\%)$ & $7(28.0)$ & $11(42.3)$ & $7(29.2)$ \\
\hline Female & $n(\%)$ & $18(72.0)$ & $15(57.7)$ & $17(70.8)$ \\
\hline \multicolumn{5}{|l|}{ Race } \\
\hline Asian & $n(\%)$ & $12(48.0)$ & $13(50.0)$ & $12(50.0)$ \\
\hline Australian aboriginal & $n(\%)$ & 0 & 0 & $1(4.2)$ \\
\hline Caucasian & $n(\%)$ & $13(52.0)$ & $13(50.0)$ & $11(45.8)$ \\
\hline \multicolumn{5}{|l|}{ Country } \\
\hline $\mathrm{AU}$ & $n(\%)$ & $13(52.0)$ & $13(50.0)$ & $12(50.0)$ \\
\hline TW & $n(\%)$ & $12(48.0)$ & $13(50.0)$ & $12(50.0)$ \\
\hline BMI $\left(\mathrm{kg} / \mathrm{m}^{2}\right)$ & Mean (SD) & $27.93(4.655)$ & $27.65(4.286)$ & $27.96(5.114)$ \\
\hline Baseline VAS score (study knee) & Mean (SD) & $6.56(1.049)$ & $6.45(1.113)$ & $6.87(1.215)$ \\
\hline Baseline WOMAC-Pain subscale score (0-4) & Mean (SD) & $1.62(0.609)$ & $1.49(0.558)$ & $1.74(0.631)$ \\
\hline Baseline WOMAC-Function subscale score (0-4) & Mean (SD) & $1.48(0.652)$ & $1.53(0.531)$ & $1.82(0.742)$ \\
\hline \multicolumn{5}{|l|}{ Knee pain } \\
\hline Bilateral & $n(\%)$ & $15(60.0)$ & $16(61.5)$ & $15(62.5)$ \\
\hline Unilateral & $n(\%)$ & $10(40.0)$ & $10(38.5)$ & $9(37.5)$ \\
\hline \multicolumn{5}{|l|}{ KL grade } \\
\hline 2 & $n(\%)$ & $9(36.0)$ & $13(50.0)$ & $9(37.5)$ \\
\hline 3 & $n(\%)$ & $16(64.0)$ & $13(50.0)$ & $15(62.5)$ \\
\hline
\end{tabular}

$A \cup$ Australia, $B M I$ body mass index, $n$ number of participants, $S D$ standard deviation, TW Taiwan

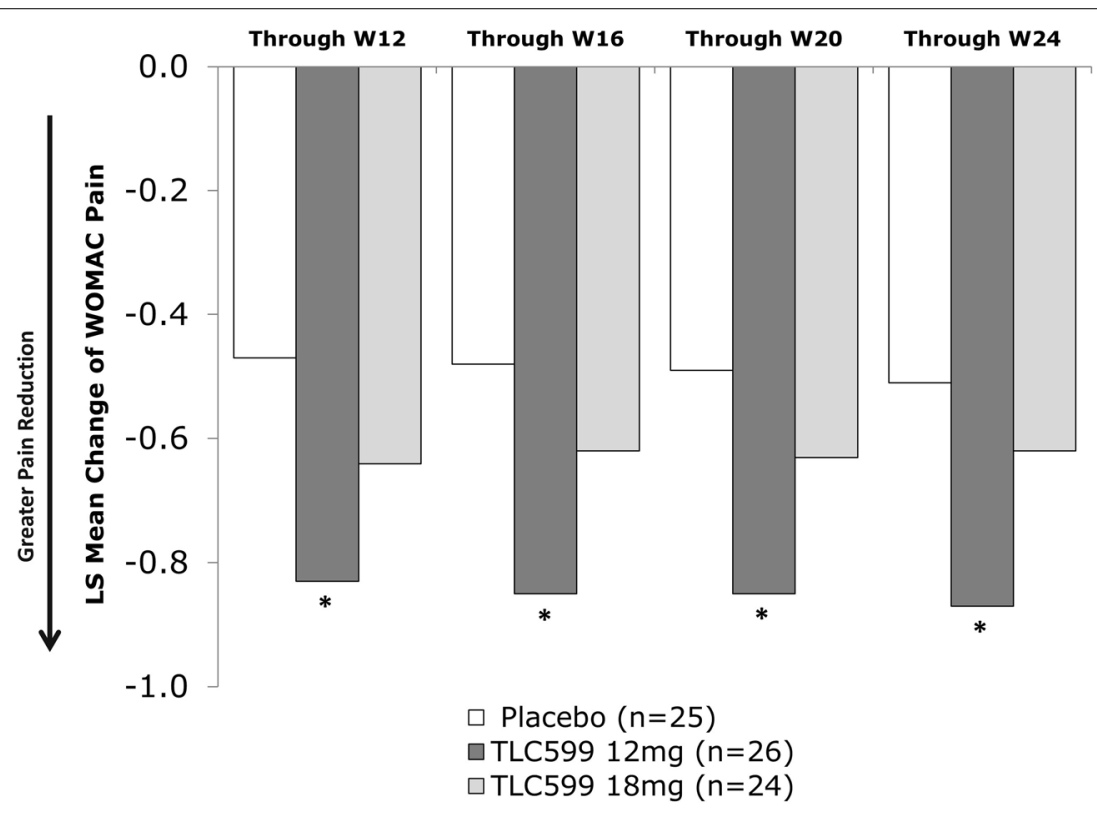

*Statistical significance $(p<0.05$ vs placebo).

Fig. 2 The mean change from baseline in WOMAC-Pain scores through scheduled visits. ${ }^{*} p<0.05$. LS, least squares 
Table 2 Statistical analysis on the mean change from baseline in WOMAC-Pain scores through scheduled visits

\begin{tabular}{|c|c|c|c|c|c|c|c|}
\hline \multirow[t]{2}{*}{ Interval } & \multirow[t]{2}{*}{ Treatment } & \multirow[t]{2}{*}{ Number } & \multirow[t]{2}{*}{ LS mean (SE) } & \multirow[t]{2}{*}{$90 \% \mathrm{Cl}$} & \multicolumn{3}{|c|}{ Difference vs placebo } \\
\hline & & & & & LS mean (SE) & $90 \% \mathrm{Cl}$ & $p$-value \\
\hline \multirow[t]{3}{*}{ D3 - W12 } & Placebo $(N=25)$ & 25 & $-0.47(0.092)$ & $(-0.619,-0.313)$ & & & \\
\hline & TLC599 $12 \mathrm{mg}(\mathrm{N}=26)$ & 26 & $-0.83(0.090)$ & $(-0.984,-0.685)$ & $-0.37(0.129)$ & $(-0.583,-0.154)$ & 0.0027 \\
\hline & TLC599 18 mg $(N=24)$ & 24 & $-0.64(0.094)$ & $(-0.794,-0.481)$ & $-0.17(0.131)$ & $(-0.390,0.047)$ & 0.0971 \\
\hline \multirow[t]{3}{*}{ D3 - W16 } & Placebo $(N=25)$ & 25 & $-0.48(0.091)$ & $(-0.633,-0.332)$ & & & \\
\hline & TLC599 12 mg ( $(N=26)$ & 26 & $-0.85(0.088)$ & $(-0.999,-0.705)$ & $-0.37(0.127)$ & $(-0.580,-0.158)$ & 0.0024 \\
\hline & TLC599 18 mg ( $(N=24)$ & 24 & $-0.62(0.093)$ & $(-0.772,-0.463)$ & $-0.13(0.129)$ & $(-0.350,0.080)$ & 0.1498 \\
\hline \multirow[t]{3}{*}{ D3 - W20 } & Placebo $(N=25)$ & 25 & $-0.49(0.091)$ & $(-0.647,-0.343)$ & & & \\
\hline & TLC599 12 mg ( $(N=26)$ & 26 & $-0.85(0.089)$ & $(-1.000,-0.704)$ & $-0.36(0.127)$ & $(-0.570,-0.145)$ & 0.0033 \\
\hline & TLC599 18 mg $(N=24)$ & 24 & $-0.63(0.093)$ & $(-0.782,-0.472)$ & $-0.13(0.130)$ & $(-0.349,0.084)$ & 0.1558 \\
\hline \multirow[t]{3}{*}{ D3 - W24 } & Placebo $(N=25)$ & 25 & $-0.51(0.092)$ & $(-0.666,-0.361)$ & & & \\
\hline & TLC599 12 mg ( $(N=26)$ & 26 & $-0.87(0.089)$ & $(-1.014,-0.718)$ & $-0.35(0.128)$ & $(-0.565,-0.139)$ & 0.0037 \\
\hline & TLC599 18 mg ( $(N=24)$ & 24 & $-0.62(0.093)$ & $(-0.780,-0.469)$ & $-0.11(0.130)$ & $(-0.328,0.106)$ & 0.1985 \\
\hline
\end{tabular}

LS means, Cls, and $p$-values were obtained from an MMRM model including factors of treatment, visit, and baseline value as fixed factors, center as a random factor, and treatment by visit as interaction terms. $C I$ confidence interval, $D$ day, LS least squares, $m / T T$ modified intent to treat, $M M R M$ mixed effect model repeat measurement, $S E$ standard error, $W$ week

$12 \mathrm{mg}$ demonstrated a statistically significantly greater reduction in pain compared to placebo at all

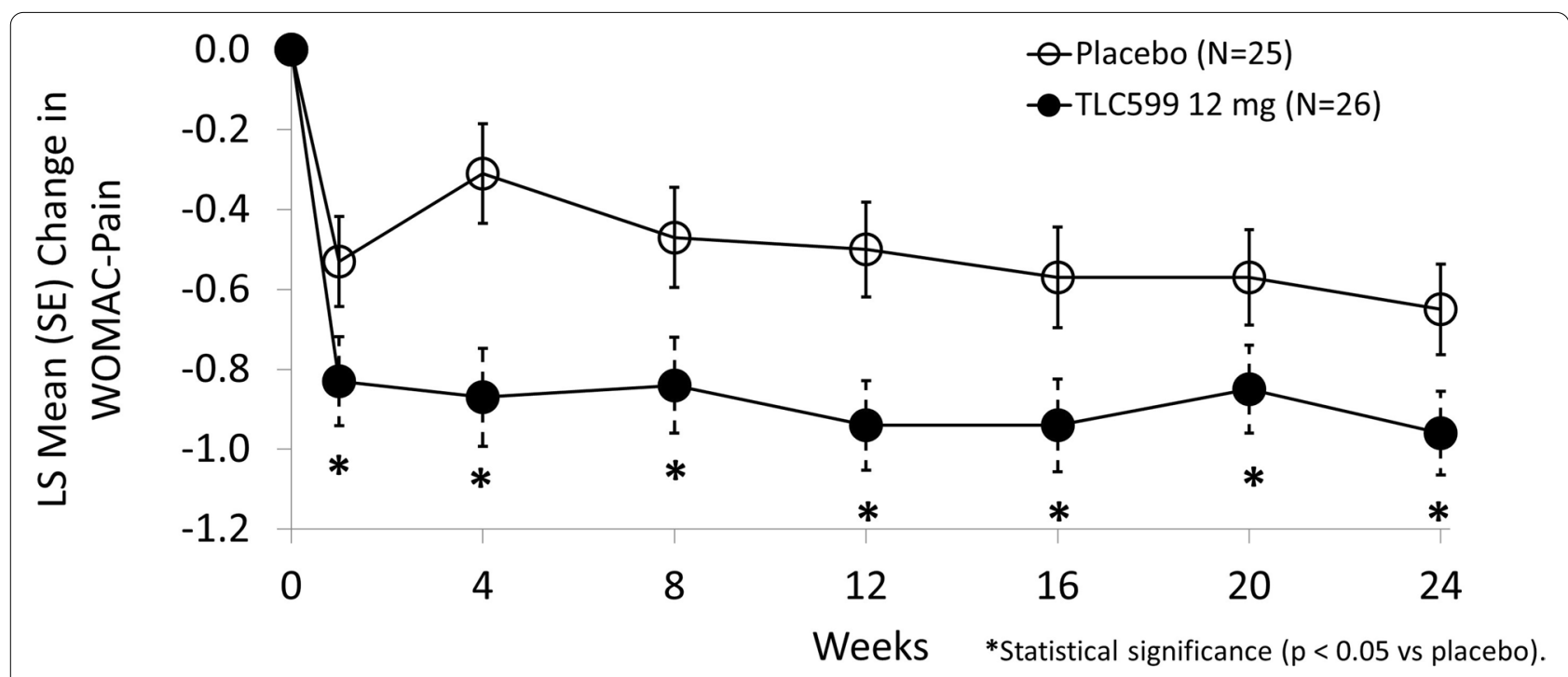

\begin{tabular}{ccc}
\hline Mean change in WOMAC Pain & \multicolumn{2}{c}{ Value (SE) } \\
\hline Week & Placebo & TLC599 $12 \mathrm{mg}$ \\
0 & 0 & 0 \\
1 & $-0.53(0.113)$ & $-0.83(0.111)$ \\
4 & $-0.31(0.125)$ & $-0.87(0.123)$ \\
8 & $-0.47(0.125)$ & $-0.84(0.120)$ \\
12 & $-0.50(0.119)$ & $-0.94(0.112)$ \\
16 & $-0.57(0.126)$ & $-0.94(0.116)$ \\
20 & $-0.57(0.119)$ & $-0.85(0.110)$ \\
24 & $-0.65(0.114)$ & $-0.96(0.105)$ \\
\hline
\end{tabular}

Fig. 3 The mean change from baseline in WOMAC-Pain scores at scheduled visits. ${ }^{*} p<0.05$. LS, least squares; SE, standard error 


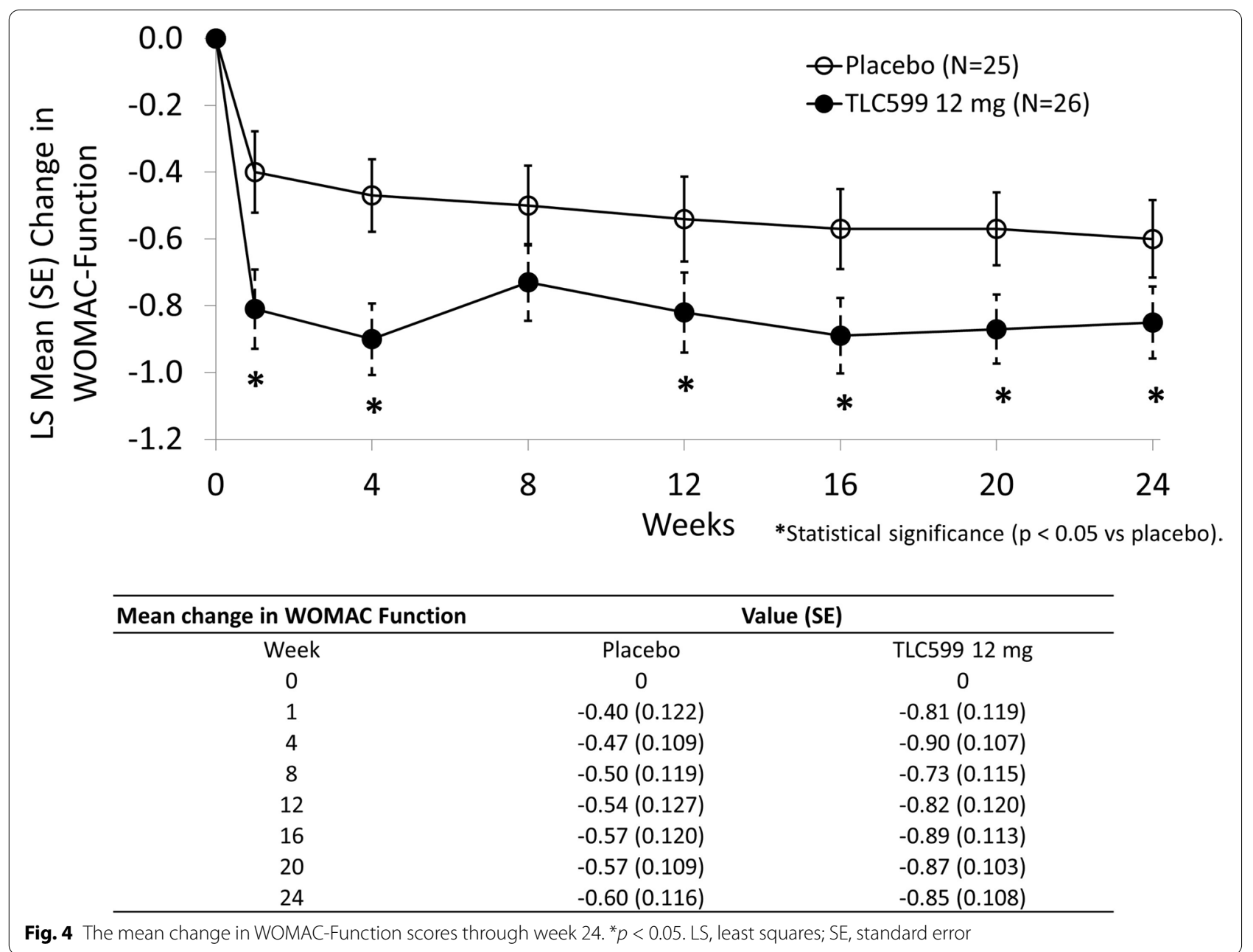

post-dosing visits (Fig. 3); those receiving TLC599 18 mg only achieved a significant reduction in pain versus placebo at week 4 .

The percentage of durable responders was significantly greater in the TLC599 $12 \mathrm{mg}$ group (56.0\%) compared with the placebo group through week $12(56.0 \%$ vs $28.6 \% ; p=0.0100)$ and through week $24(52.0 \%$ vs $22.2 \% ; p=0.0143)$; differences between TLC599 $18 \mathrm{mg}$ and placebo groups did not reach statistical significance.

Improvement in physical function with TLC599 was assessed using the WOMAC-Function subscale. Participants receiving one injection of TLC599 $12 \mathrm{mg}$ displayed statistically significantly greater improvement in function than those treated with placebo at all time points except at week 8 (Fig. 4). TLC599 $18 \mathrm{mg}$ did not demonstrate significantly greater improvement in function in comparison with placebo at any time point.

In the interval analyses of reduction in pain through week 24 utilizing the VAS, results were similar to those of the WOMAC-Pain. Concordant with the WOMACPain results at the individual scheduled visit, TLC599 12 mg-treated participants also demonstrated a significantly greater reduction in pain compared to placebo at all time points, including week 24 visit (LS mean difference $=$ $-1.38, p=0.0319$ ) (Fig. 5). TLC599 $18 \mathrm{mg}$ only demonstrated a significantly greater reduction in VAS pain in comparison with placebo at week 8 .

Regarding the life of quality assessment utilizing EQ-5D questionnaires, improvements with both TLC599 group treatment groups were not statistically superior to placebo at most time points (data not shown).

The use of acetaminophen during the study was less than the placebo group in both of the TLC599-treated groups throughout 24 weeks. Acetaminophen use among the TLC599 12 mg-treated participants was significantly lower than that of the placebo group at most time points through week 20 (Fig. 6). 


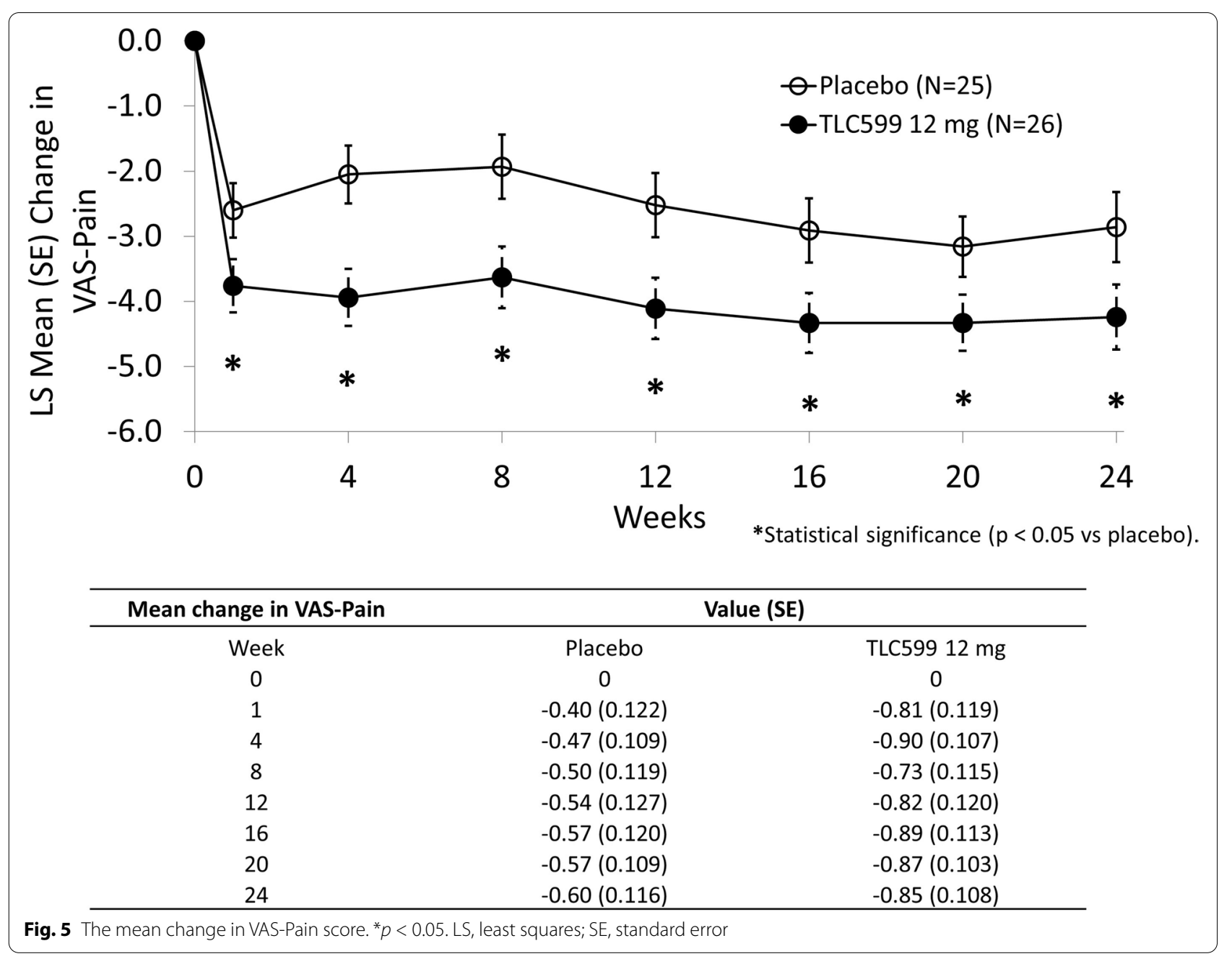

\section{Safety}

Overall, 18 (69.2\%) participants in the TLC599 12 $\mathrm{mg}$ group, 20 (83.3\%) participants in the TLC599 $18 \mathrm{mg}$ group, and 17 (68.0\%) participants in the placebo group reported at least 1 treatment-emergent $\mathrm{AE}$ (TEAE). Frequent adverse events are shown in Table 3. Treatment-emergent adverse events judged to be at least possibly related to the study treatment were reported in $26.9 \%$ of the participants treated with TLC599 $12 \mathrm{mg}$ and $45.8 \%$ of the participants treated with TLC599 $18 \mathrm{mg}$ (compared to 16\% with placebo). Most TEAEs were mild or moderate in severity. There was 1 serious AE of clear cell renal cell carcinoma, reported for a participant in the TLC599 $12 \mathrm{mg}$ group, which was judged not related to study treatment. No deaths or TEAEs leading to participant discontinuation were reported in this study.
Laboratory abnormalities of blood cortisol levels at routine study visits were reported as TEAEs with coded terms of "cortisol decreased" in 8 participants and "glucocorticoid deficiency" in 4 participants (all in TLC599treated subjects). All events were resolved, typically by the next scheduled laboratory assessment, and were not accompanied by associated signs and symptoms that might be attributed to hypocortisolism. No other notable laboratory trends were found in this study, and no deleterious effects on the study knees were observed by MRI in TLC599 and placebo treatment groups.

\section{Discussion}

The modest duration of corticosteroids leads to a need for repeat injection for patients with chronic joint pain due to knee OA. However, the potential chondrotoxicity of common corticosteroid drugs such as triamcinolone 


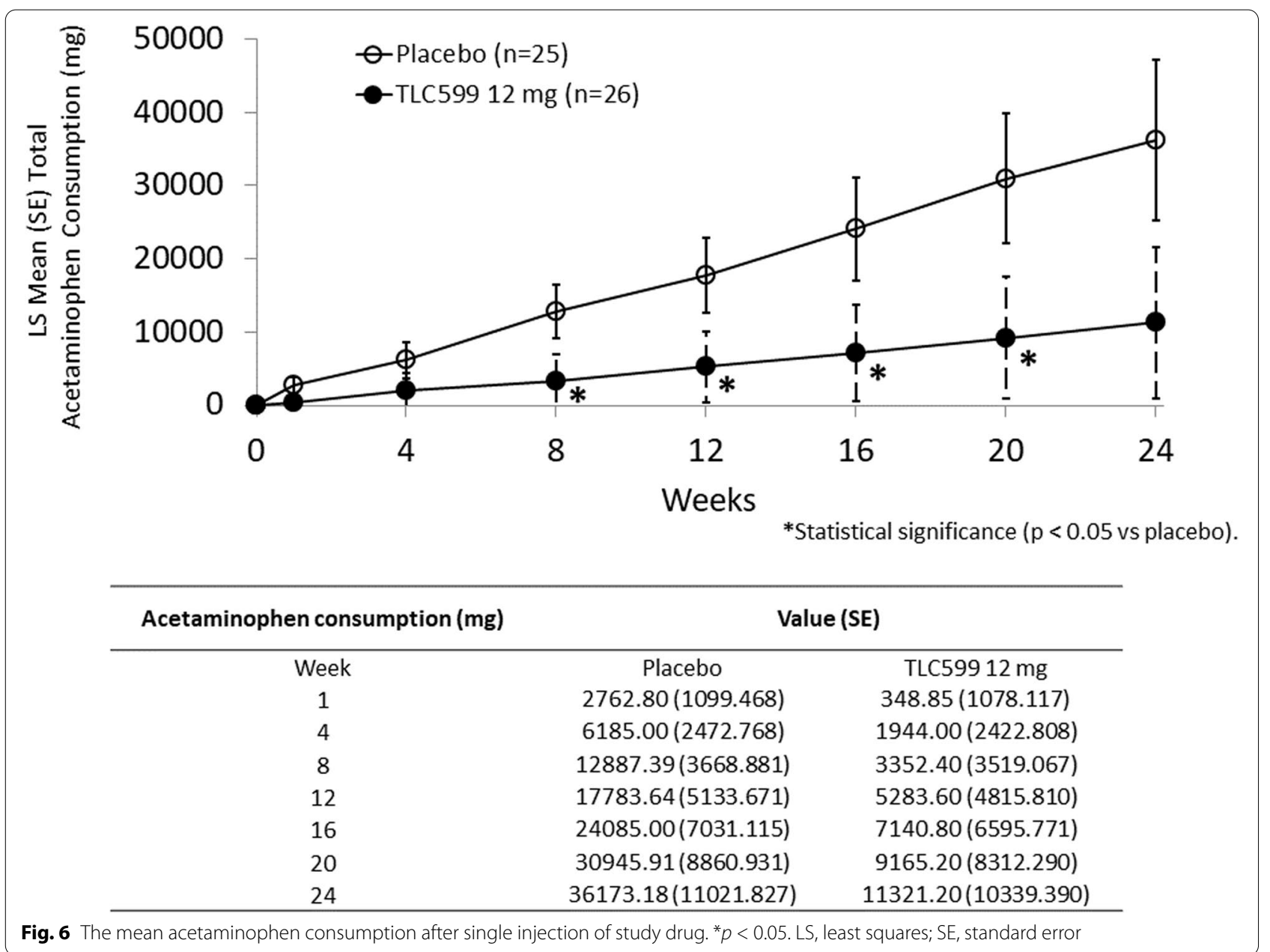

acetonide also limits the frequency of its injection and restricts its clinical benefit for long-term pain management [12]. The development of the water-soluble corticosteroid dexamethasone sodium phosphate with both immediate-release and sustained-release profiles in the liposomal formulation is anticipated to solve this problem by providing satisfactory and durable pain relief and reducing the injection frequency. Notably, in toxicology studies in dogs and rabbits, neither cartilage damage nor proteoglycan loss was observed after repeated dosing with TLC599 [14].

The results from this clinical study have demonstrated TLC599's long-acting efficacy in comparison with placebo. In contrast to another approved steroid product of sustained-release formulation [15], the current study has shown the duration of pain control of a single TLC599 injection could be up to week 24 without a decline in effect. A larger and well-designed pivotal study (Clini calTrials.gov identifier: NCT04123561) is currently ongoing to confirm this efficacy profile. It was reported that reductions in chronic pain intensity in individuals of at least 30\% appear to reflect at least moderate clinically important differences, and it is recommended that the percentages of patients responding with this degree of pain relief be reported in clinical trials of chronic pain treatments [16]. The dose level of TLC599 $12 \mathrm{mg}$ in this study demonstrated a defined durable reduction $(>30 \%$ reduction at each study visit) in pain and improvement in physical function.

Acetaminophen is a mild analgesic with little meaningful clinical benefit and real risks of harm, and the American Academy of Orthopedic Surgeons suggests no more than $3000 \mathrm{mg}$ per day to minimize its risk of liver damage [17-19]. The current study allowed acetaminophen as the only rescue medication during the study period, and the TLC599 $12 \mathrm{mg}$ group was observed to consume significantly less acetaminophen than the placebo group, as assessed at most visits. TLC599 has the potential to reduce the need for oral medication use in the setting where opioids are frequently resorted to for pain control. 
Table 3 Frequent (> 5\%) treatment-emergent adverse events (safety population)

\begin{tabular}{|c|c|c|c|}
\hline AE category & Placebo $(N=25)$ & TLC599 $12 \mathrm{mg}(N=26)$ & $\begin{array}{l}\text { TLC599 } 18 \\
\mathrm{mg}(N= \\
24)\end{array}$ \\
\hline Any adverse event & $17(68 \%)$ & $18(69 \%)$ & $20(83 \%)$ \\
\hline Headache & $4(16 \%)$ & $3(12 \%)$ & $6(25 \%)$ \\
\hline Nasopharyngitis & $3(12 \%)$ & $4(15 \%)$ & $1(4 \%)$ \\
\hline Cortisol decreased & 0 & $2(8 \%)$ & $6(25 \%)$ \\
\hline Upper respiratory tract infection & $2(8 \%)$ & $1(4 \%)$ & $4(17 \%)$ \\
\hline Arthralgia & $3(12 \%)$ & $1(4 \%)$ & $2(8 \%)$ \\
\hline Glucocorticoid deficiency & 0 & $3(12 \%)$ & $1(4 \%)$ \\
\hline Bronchitis & $3(12 \%)$ & 0 & 0 \\
\hline Cough & 0 & 0 & $2(8 \%)$ \\
\hline Diarrhea & 0 & $3(12 \%)$ & 0 \\
\hline Dyspepsia & 0 & $2(8 \%)$ & 0 \\
\hline Lipase increased & $2(8 \%)$ & 0 & $1(4 \%)$ \\
\hline Toothache & $1(4 \%)$ & 0 & $2(8 \%)$ \\
\hline Urinary tract infection & $1(4 \%)$ & $2(8 \%)$ & 0 \\
\hline Chronic kidney disease & $2(8 \%)$ & 0 & 0 \\
\hline Injury & $2(8 \%)$ & 0 & 0 \\
\hline
\end{tabular}

In contrast to the TLC599 $12 \mathrm{mg}$ dose, the $18 \mathrm{mg}$ dose did not demonstrate statistically greater pain reduction and function improvement over placebo. The reasons for this are unknown and may be attributable to the small sample size in this study. However, this finding was consistent with an in-vitro drug release study of TLC599 at different dose levels $(5.1,8,18$, and $36 \mathrm{mg})$ in artificial synovial fluid, in which the rate of DSP release was greatly reduced at doses of $18 \mathrm{mg}$ and higher (unpublished data), suggesting that doses less than $18 \mathrm{mg}$ may be optimal for both immediate and sustained effect of the drug. As TLC599 $12 \mathrm{mg}$ showed greater efficacy compared to TLC599 $18 \mathrm{mg}, 12 \mathrm{mg}$ can be considered the dose of choice for further clinical investigation of TLC599 in treating OA knee pain.

TLC599 was shown to be well tolerated in the current study. Although a reduction in cortisol was observed in a portion of participants at the beginning of the study (less in the TLC599 $12 \mathrm{mg}$ group than in the $18 \mathrm{mg}$ group), such transient cortisol reduction is a well-described physiologic response after IA cortisol injections [20]. The pharmacodynamic reduction in cortisol typically evidenced recovery after 1 week and was not associated with any adverse signs or symptoms. The safety profile seen in participants who received TLC599 was generally consistent with that expected in an older population of OA patients receiving IA corticosteroid injections.

\section{Conclusions}

The results of the current study suggest that TLC599 provides durable pain relief and functional improvement while maintaining a satisfactory safety profile for a time period well beyond that of current treatment options. Replication in a larger phase III study with adequate ethnic diversity will be required to confirm the reported findings.

\section{Abbreviations}

Cl: Confidence interval; DSP: Dexamethasone sodium phosphate; ECG: Electrocardiogram; EQ-5D: EuroQol-5 Dimension; HbA1c: Hemoglobin A1c; IEC: Independent ethics committee; IRB: Institutional review board; IWRS: Interactive Web Response System; KL grade: Kellgren-Lawrence grade; LS: Least squares; mITT: Modified intent-to-treat; MMRM: Mixed-effects model for repeated measures; MRI: Magnetic resonance imaging; NSAID: Non-steroidal anti-inflammatory drugs; OA: Osteoarthritis; OARSI: Osteoarthritis Research Society International; PL: Phospholipid; SAS: Statistical Analysis System; SE: Standard error; TEAE: Treatment-emergent adverse event; VAS: Visual analog scale; WOMAC: Western Ontario and McMaster Universities Arthritis Index.

\section{Acknowledgements}

The authors would like to acknowledge all the participants and investigators who participated in the TLC599A2003 trial. In addition, the authors would like to acknowledge Weenee Jao and Renee Chih for the medical advice and support of the study conduct.

\section{Authors' contributions}

All authors participated in the interpretation of the study results and in the critical revision and approval of the final version of the manuscript. DH, CW, TT, and SS contributed to the study conception and design. CC, JW, and HL contributed to the acquisition of the study results. CB, CW, WC, and SS conducted the data analysis and manuscript drafting.

Funding

The current study was fully sponsored by the Taiwan Liposome Company, Ltd. 


\section{Availability of data and materials}

The datasets generated and/or analyzed during the current study are not publicly available due to commercial confidentiality reasons but are available from the corresponding author on reasonable request.

\section{Declarations}

\section{Ethics approval and consent to participate}

The study protocol, all study protocol amendments, written study participant information, informed consent form, Investigator's Brochure, and any other relevant documents were reviewed and approved by an independent ethics committee (IEC) or institutional review board (IRB) at each study center. The study was conducted in accordance with the approved protocol and the ethical principles derived from international guidelines, including the Declaration of Helsinki (2013), International Council for Harmonisation, Good Clinical Practice Guidelines, and applicable laws and regulations. The ethical committees associated with this study include the following: Cheng Hsin General Hospital IRB ((570)105B-39), Taipei Medical University-Joint IRB (N201609008), Kaohsiung Medical University Chung-Ho Memorial Hospital IRB (KMUHIRBF(II)-20160109), Shin Kong Wu Ho-Su Memorial Hospital IRB (20170202C), China Medical University and Hospital Research Ethics Committee (HREC) (CMUH106-REC3-056), Show Chwan Memorial Hospital IRB (1060212), Chung Shan Medical University Hospital IRB (CS2-17055), Taichung Veterans General Hospital IRB (SC17266-B), Northern Sydney Local Health District HREC (RESP/16/296), Bellberry Human Research Ethics Committee (2016-10-748), and Northern Sydney Local Health District HREC (RESP/16/296).

\section{Consent for publication}

Not applicable.

\section{Competing interests}

David J Hunter provides consulting advice to Merck Serono, Pfizer, Lilly, and TLCBio. James Cheng-Chung Wei received research grant, speaker fee, or advisor board from Pfizer, Abbott, Abbvie, Bristol-Myers Squibb, Eli Lilly, JNJ, UCB, MSD, GSK, Chugai, Roche, Celgene, Sanofi-Aventis, and Novartis. Sheue-Fang Shih, Carl Brown, Tien-Tzu Tai, and Wing Chia-Ming Chuang are employees and shareholders of the Taiwan Liposome Company. Chih-Feng Wu was an employee of Taiwan Liposome Company during the trial conduct but was no longer in the company at the time of this manuscript submission. The rest of the authors declare that they have no competing interests.

\section{Author details}

${ }^{1}$ Institute of Bone and Joint Research, Kolling Institute, University of Sydney, Sydney, Australia. ${ }^{2}$ Rheumatology Department, Royal North Shore Hospital, St Leonards, NSW 2065, Australia. ${ }^{3}$ Division of Allergy, Immunology and Rheumatology, Department of Internal Medicine, School of Medicine, College of Medicine, Taipei Medical University, Taipei City, Taiwan. ${ }^{4}$ Division of Rheumatology, Immunology and Allergy, Department of Internal Medicine, Taipei Medical University Hospital, Taipei City, Taiwan. ${ }^{5}$ Institute of Medicine, Chung Shan Medical University, Taichung City, Taiwan. ${ }^{6}$ Division of Allergy, Immunology and Rheumatology, Chung Shan Medical University Hospital, Taichung City, Taiwan. ${ }^{7}$ Graduate Institute of Integrated Medicine, China Medical University, Taichung, Taiwan. ${ }^{8}$ Department of Medicine, Cheng Hsin General Hospital, Taipei City, Taiwan. ${ }^{9}$ School of Medicine, National Yang-Ming University, Taipei City, Taiwan. ${ }^{10}$ Taiwan Liposome Company, Ltd., 2F, No. 3 Yuanqu St., Nangang Dist., Taipei City 115, Taiwan.

Received: 2 September 2021 Accepted: 6 February 2022

Published online: 21 February 2022

\section{References}

1. Kloppenburg M, Berenbaum F. Osteoarthritis year in review 2019: epidemiology and therapy. Osteoarthritis Cartilage. 2020;28(3):242-8. https://doi. org/10.1016/j.joca.2020.01.002.

2. Neogi T. The epidemiology and impact of pain in osteoarthritis. Osteoarthritis Cartilage. 2013;21(9):1145-53.
3. Osteoarthritis: A serious disease. https://www.oarsi.org/sites/default/ files/docs/2016/oarsi_white_paper_oa_serious_disease_121416_1.pdf. Accessed 02 Sept 2021.

4. Newberry SJ, FitzGerald J, SooHoo NF, Booth M, Marks J, Motala A, Apaydin E, Chen C, Raaen L, Shanman RSP. Treatment of osteoarthritis of the knee: an update review. Rockville (MD): Agency for Healthcare Research and Quality (US); 2017 May. (Comparative Effectiveness Reviews, No. 190.). https://www. ncbi.nlm.nih.gov/books/NBK447543/. Accessed 02 Sept 2021.

5. Deshpande BR, Katz JN, Solomon DH, Yelin EH, Hunter DJ, Messier SP, et al. Number of persons with symptomatic knee osteoarthritis in the US: impact of race and ethnicity, age, sex, and obesity. Arthritis Care Res. 2016;68:1743-50

6. Lespasio MJ, Nicolas SP, Elaine Husni M, Muschler GF, Guarino AJ, Mont MA. Perm J. 2017:21:16-183.

7. Nelson AE, Allen KD, Golightly YM, Goode AP, Jordan JM. A systematic review of recommendations and guidelines for the management of osteoarthritis: the Chronic Osteoarthritis Management Initiative of the U.S. Bone and Joint Initiative. Semin Arthritis Rheum. W.B. Saunders. 2014;43:701-12.

8. Lee B, Sodhi N, Anis HK, Ehiorobo JO, Mont MA. Injection alternatives for the management of knee osteoarthritis pain. Surg Technol Int. 2019;15(34):513-9.

9. Jüni P, Hari R, Rutjes AWS, Fischer R, Silletta MG, Reichenbach S, et al. Intraarticular corticosteroid for knee osteoarthritis. Cochrane Database Syst Rev. 2015;10:CD005328.

10. Zhang W, Robertson WB, Zhao J, Chen W, Xu J. Emerging trend in the pharmacotherapy of osteoarthritis. Front Endocrinol (Lausanne). 2019;2(10):431. https://doi.org/10.3389/fendo.2019.00431.

11. Zuber TJ. Knee joint aspiration and injection. Am Fam Physician. 2002:66:1497-1500+1503

12. McAlindon TE, LaValley MP, Harvey WF, Price LL, Driban JB, Zhang M, et al. Effect of intra-articular triamcinolone vs saline on knee cartilage volume and pain in patients with knee osteoarthritis: a randomized clinical trial. JAMA. 2017;317(19):1967-75.

13. https://www.tlcbio.com/en-global/technology/index/sustained-release/ bioseizer. Accessed 12 Nov 2021.

14. Yu WN, Wu MJ, Chang PC, Shih SF. Cartilage damage and synovial toxicokinetic study of a sustained release liposomal formulation of dexamethasone sodium phosphate (TLC599) following intra-articular injection in healthy dogs and rabbits. Osteoarthr Cartil. 2019;27(1):S161-S162.

15. Conaghan PG, Hunter DJ, Cohen SB, Kraus VB, Berenbaum F, Lieberman JR, et al. Effects of a single intra-articular injection of a microsphere formulation of triamcinolone acetonide on knee osteoarthritis pain. J Bone Joint Surg Am. 2018;100(8):666-77. https://doi.org/10.2106/JBJS.17.00154.

16. Dworkin RH, Turk DC, Wyrwich KW, Beaton D, Cleeland CS, Farrar JT, et al. Interpreting the clinical importance of treatment outcomes in chronic pain clinical trials: IMMPACT recommendations. J Pain. 2008;9(2):105-21.

17. Holubek WJ, Kalman S, Hoffman RS. Acetaminophen-induced acute liver failure: results of a United States multicenter, prospective study. Hepatology. 2006;43(4):880 author reply 882.

18. Machado GC, Maher CG, Ferreira PH, Pinheiro MB, Lin CWC, Day RO, et al. Efficacy and safety of paracetamol for spinal pain and osteoarthritis: systematic review and meta-analysis of randomised placebo controlled trials. BMJ. 2015:31(350):h1225.

19. Roberts E, Delgado Nunes V, Buckner S, Latchem S, Constanti M, Miller P, et al. Paracetamol: not as safe as we thought? A systematic literature review of observational studies. Ann Rheum Dis. 2016;75(3):552-9.

20. Lazarevic MB, Skosey JL, Djordjevic-Denic G, Swedler WI, Zgradic I, Myones $B L$. Reduction of cortisol levels after single intra-articular and intramuscular steroid injection. Am J Med. 1995;99(4):370-3.

\section{Publisher's Note}

Springer Nature remains neutral with regard to jurisdictional claims in published maps and institutional affiliations. 